\title{
Jahrestagung des Deutschen Ethikrats 2016: Zugriff auf das menschliche Erbgut. Neue Möglichkeiten und ihre ethische Beurteilung
}

\author{
Berlin, 22. Juni 2016
}

\section{Christiane Burmeister $\cdot$ Robert Ranisch}

Online publiziert: 19. Oktober 2016

(C) Springer-Verlag Berlin Heidelberg 2016

Mit dem Titel „Zugriff auf das menschliche Erbgut - Neue Möglichkeiten und ihre ethische Beurteilung "1 widmete der Deutsche Ethikrat am 22. Juni im Ellington Hotel Berlin seine Jahrestagung einem der gegenwärtig meistdiskutierten Themen der Bio- und Medizinethik. Seitdem jüngere molekularbiologische Verfahren wie CRISPR-Cas9 überraschend effiziente Eingriffe in Gene und Erbgut ermöglichen, häufen sich bemerkenswerte Forschungsereignisse und -visionen von genetischer Veränderung sowohl pflanzlicher als auch tierischer Zellen, und mit ihnen die Veröffentlichung von Stellungnahmen zum verantwortungsbewussten Umgang mit dieser Technologie. ${ }^{2}$ Die in diesem Zusammenhang frappierendsten Vorstöße unternahm kürzlich eine chinesische Forschergruppe mit der Manipulation des Erbgutes (nichtlebensfähiger) menschlicher Embryonen (Liang et al. 2015).

Entsprechend überraschte nicht, dass auch der kürzlich neu zusammengesetzte Ethikrat dem wissenschaftlich-öffentlichen Austausch über diese Entwicklungen ein

\footnotetext{
1 Folien und Mitschnitte der Tagung, online siehe: Deutscher Ethikrat (2016).

2 Exemplarisch seien hier die Stellungnahme zum Genome Editing der Nationalen Akademie der Wissenschaften Leopoldina genannt, wie auch die Analyse der Interdisziplinären Arbeitsgruppe Gentechnologiebericht der Berlin-Brandenburgischen Akademie der Wissenschaften. Für den US-amerikanischen Raum ist insbesondere die Human Gene-Editing Initiative der National Academy of Sciences sowie der National Academy of Medicine hervorzuheben.

C. Burmeister, M.A. · R. Ranisch, M.A. $(\bowtie)$

Global Applied Ethics Institute (GAEI), c/o Ethikzentrum Jena, Friedrich-Schiller-Universität Jena, Zwätzengasse 3, 07743 Jena, Deutschland

E-Mail: robert.ranisch@gaei.org

C. Burmeister, M.A. · R. Ranisch, M.A.

Internationales Zentrum für Ethik in den Wissenschaften (IZEW), Eberhard Karls Universität Tübingen, Tübingen, Deutschland
} 
groß angelegtes Forum bot. Wie der neue Vorsitzende Peter Dabrock in seiner Eröffnungsrede erklärte, sei man ,,an einem Punkt, an dem man sich zu den Möglichkeiten von CRISPR-Cas nicht nicht verhalten kann“. Gleichwohl bemerkenswert war die im Konferenzthema fixierte Fokussierung auf die Anwendungsfelder der „Genomchirurgie“ im humanen Bereich. Zwar sind die Erwartungen an eine lebhafte Kontroverse zu diesem Thema gut nachvollziehbar. Ob es sich hierbei aber um die bedrängendste ethische Fragestellung bei den gegenwärtig verhandelten Verfahren handelt, wurde mit Verweis auf bereits erfolgendes Gene Editing an Nutzpflanzen, Pilzen und Tieren sowie Überlegungen zu Gene Drive-basierten Veränderungen ganzer Populationen, wie gegenwärtig im Zusammenhang mit der Eindämmung des Zika-Virus diskutiert wird, kritisch hinterfragt.

Als treffend erwiesen sich nichtsdestoweniger Dabrocks einleitende Worte, CRISPR-Cas habe ,,alles Zeug, wie ein Gespenst zu wirken“; jenes Gespenst, das im Sinne Marx' und Engels' umhergeht, an der Schwelle zwischen Leben und Nicht-Leben agiert, Fallen des Irrationalen stellt und eine etablierte Ordnung durcheinander bringt. Diese Metapher aufgreifend kann bemerkt werden, dass im Verlauf der Tagung nicht allein das Gespenst zum Gegenstand der Reflexionen wurde, sondern auch die Unwirklichkeit, die es umgibt.

Nachdem zu Beginn anhand von Grundlagenvorträgen zum Sachstand der „Genomchirurgie“ (Jörg Vogel, Würzburg und Karl Welte, Tübingen) ein naturwissenschaftliches bzw. medizinisches Licht auf den vermeintlichen Spuk geworfen wurde, förderte bereits der dritte Vortrag ein Beispiel für die von Dabrock erwähnte Konfusion einer etablierten Ordnung zutage: So errichtet das deutsche Embryonenschutzgesetz (ESchG) von 1990 nach Jochen Taupitz (Mannheim) entgegen dem ersten Eindruck und womöglich seinem eigenen Anspruch keine lückenlose Schranke vor Keimbahninterventionen. Zwar gilt das in $\S 5$ verankerte grundsätzliche Verbot der künstlichen Veränderung menschlicher Keimbahnzellen. ${ }^{3}$ Jedoch enthält Absatz 4 entscheidende Ausnahmen bezüglich der Manipulation von Keimbahnzellen, wenn deren Übertragung auf Embryo, Fötus oder Mensch sowie die Entstehung eines neuen Menschen ausgeschlossen ist. Für veränderte Keimzellen gilt wiederum lediglich, dass sie nicht zur Befruchtung gebracht werden dürfen. Damit seien auch in Deutschland entsprechende In-vitro-Versuche an Zellen nicht verboten.

Weitere Ausnahmetatbestände stellen nach Taupitz therapeutische Maßnahmen dar, die mögliche Keimbahnveränderungen lediglich in Kauf nehmen, aber nicht zum Ziel haben. Hier habe das konkrete Wohl geborener wie ungeborener Individuen Vorrang vor der ungewissen Gefahr für zukünftige Individuen. Hinzu käme „eine Reihe erstaunlicher Lücken“ im ESchG, wie das Fehlen eines Verbots der Keimbahnintervention für das Ersetzen des Kerns einer Eizelle durch den Kern einer anderen. ${ }^{4}$ Ebenso würde $§ 5$ ESchG nur rein menschliche Keim(bahn)zellen

\footnotetext{
3 Jenen Vorläuferzellen also, aus denen sich Ei- und Samenzellen (Keimzellen) entwickeln.

4 Grund dafür sei, dass es sich bei einer entkernten Eizelle nicht mehr um eine vollständige und damit um eine Keimzelle im Sinne des Gesetzes handelt. Die Erbinformation wird streng genommen nicht verändert, sie wird schlicht ausgetauscht - ein Tatbestand, der nach Taupitz und angeblich vielen anderen Juristen vom ESchG nicht erfasst wird. Gleiches gilt für die Ersetzung eines Zellkerns durch den Kern einer somatischen Zelle (Dolly-Methode) - zumal, wenn im Anschluss keine Befruchtung stattfindet.
} 
erfassen, aber nicht Fälle des Kerntransfers zwischen tierischen und menschlichen Eizellen. Sollte es ferner, wie die Forschungsbemühungen um induzierte pluripotente Stammzellen (iPS-Zellen) verheißen, künftig möglich sein, Keimzellen aus beliebigen somatischen Zellen herzustellen und zur Befruchtung zu führen, sei auch dies nicht gesetzeswidrig. Ungeachtet aller ethischer Kontroversen bringe das ESchG gegen diese vor einem Vierteljahrhundert noch unvorhersehbare Technologie keine strafbewehrten Mittel auf. Der möglichen Versuchung zu unterliegen, das ESchG so zu interpretieren, als impliziere es ein Verbot dieser Praktiken, sei wiederum nicht möglich, da es als Strafgesetz nicht über seinen Wortlaut hinaus ausgelegt werden dürfe.

Was Taupitz' Interpretationen bereits andeuten, wird in der Gesetzesbegründung explizit: So war es nicht Absicht seiner Verfasser, Keimbahninterventionen an sich zu unterbinden, sondern mit ihnen verbundene Gefahren für zukünftig geborene Menschen zu verhindern. Bereits aus diesem Grund lässt sich nach Taupitz dafür argumentieren, dass die kontrovers rezipierten chinesischen Versuche auch in Deutschland nicht verboten sind, da hier nicht-lebensfähige menschliche Embryonen verwendet wurden. Risikobedenken würden zudem nicht mehr greifen, wenn Keimbahninterventionen in Zukunft hinreichend sicher durchführbar wären. Mit seinen anschließenden Auslegungen von Verfassungsrecht, Menschen- und Gattungswürde wies Taupitz gleichsam seine ethische Perspektive aus, nach der zwar die mangelnde Anwendungssicherheit des Genome Editings für dessen Nichtzulässigkeit aus Vorsichtsgründen spricht. Falls das Vorsichtsargument jedoch entfällt, sollte im Hinblick auf die konkreten Träger schwerer genetischer Krankheiten das für deren Leidensfreiheit oder -minderung Machbare getan werden. Als tatsächliches Problem benannte Taupitz hier die notwendige Indikationsstellung für einen Keimbahneingriff, die zwei kontroverse Grenzziehungen erfordert: zum einen die zwischen jenen Erbkrankheiten, deren Schweregrad eine Intervention rechtfertigen und solchen, die nicht das Ziel dieser Eingriffe sind; zum anderen die Grenzziehung zwischen Therapie und Enhancement. Dass diese Unterscheidungen nicht einfach sind, bedeute wiederum nicht, dass sie nicht getroffen werden sollten.

Das hier entfaltete und zuvor schon von Vogel und Welte genannte Vorsichtsargument wurde in vielen der nachfolgenden Vorträge aufgegriffen. Auch wenn sich die jeweiligen Schwerpunktsetzungen auf Risiken und Chancen der Genomchirurgie ebenso wie die Forderungen nach Art und Umfang eines möglichen Moratoriums erwartungsgemäß unterschieden, war doch erstaunlich, dass kategorische Bedenken gegenüber zukünftiger Keimbahnintervention in den Hintergrund traten. Die grundsätzliche Haltung „Hände weg von der Keimbahn“, die noch die Gentechnik-Debatten der späten 1990er-Jahre prägte (Hein), wich damit gleichsam der Verhandlung hypothetischer Verbote der Keimbahnveränderung.

Selbst Wolfgang Hubers (Berlin) eng an Habermas orientierter Vortrag brachte Einwände kategorischer Natur nur gegen gentechnische Eingriffe ,im Dienst der Perfektion“, nicht gegen solche ,im Dienst des Heilens“ hervor. Dass er im Fall der Keimbahn auch für therapeutische Eingriffe einen Verzicht fordert, schien im Licht des ,weltanschaulich imprägnierten“ Nichtschadensprinzips vielmehr den Risikound Unsicherheitsfaktoren generationsübergreifender Wirkungen geschuldet. Wo es wiederum um die Menschheit als Gattungswesen (Habermas) oder als Kommuni- 
kationsgemeinschaft (Apel) geht, komme es auf die Vermeidung der „Selbstaufhebung“ an, für die präzise Folgenabschätzungen probates Mittel seien. In ähnlicher Weise unterschieden auch Bischof Martin Hein (Kurhessen-Waldeck) und Eberhard Schockenhoff (Freiburg i. B.) zwischen therapeutischen Keimbahninterventionen im „,berechtigen Interesse von Betroffenen“ (Hein), welche unter bestimmten Bedingungen zulässig sein mögen, und „Verbesserungszielen der positiven Eugenik“ (Schockenhoff).

Wenngleich also alle genannten Redner den Wunsch nach einer ,unterscheidungssensiblen Diskussion“ (Dabrock) beherzigten und stets somatische von Keimbahneingriffen differenzierten, schien für sie nicht diese, sondern vielmehr die Trennlinie zwischen Therapie und Enhancement das zentrale Bewertungskriterium zu sein. Aufschlussreich waren in diesem Zusammenhang die Ausführungen Dieter Birnbachers (Düsseldorf) zum Vertrauensvorschuss der Natürlichkeit, welche diese ,zutiefst verfängliche“ Kategorie in der gegenwärtigen Verhandlung um das Thema Enhancement genieße. Zwar sei Natürlichkeit ein „schlechtes Argument“ gegen die Genmanipulation. Zugleich aber könnten entsprechende Intuitionen fruchtbar gemacht werden, da sie in sensiblen Handlungsfeldern wie der Reproduktion ein legitimes Bedürfnis nach Rückzugsräumen der „Nicht-Rationalität“ zum Ausdruck brächten und vor einem moralischen Perfektionismus sowie vor Überforderung schützten.

Die verbreitete Eingrenzung des „Schreckgespensts“ auf eine Vision gentechnischer Optimierung blieb keineswegs der einzige Umgang mit dem Thema. Mit den anschließenden Streitgesprächen rückte zunehmend die Frage ins Zentrum, ob es sich bei der bloßen Pro-/Kontra-Argumentation verschiedener Anwendungsziele und -effekte um allzu grob vereinfachende Darstellungen einer komplexen Realität handelt. Bis hierhin hatten bereits zahlreiche Publikumsfragen den Fokus auf die Anwendungspotenziale von CRISPR-Cas hinterfragt und die Gefahren einer Rückkehr zu genetischen Reduktionismen angemahnt.

Wie Andrea Esser (Jena) dann am Nachmittag betonte, erschöpft sich eine ethische Beurteilung des Genome Editing nicht in seiner aktspezifischen Dimension, sondern umfasse auch komplexe Wechselwirkungen mit dem sozialen System. Ihr Hauptaugenmerk lag hier auf einem markanten Sprach- und Bildgebrauch insbesondere im medial vermittelten Diskurs: Wenn Experimente an Mäusen mit Bildern von Menschen illustriert werden, wenn Hände in die Doppelhelix greifen (wie im Fall des Werbeplakates der Jahrestagung) oder anderorts vom „Ausradieren“ die Rede ist, dann zeigt sich, so Esser, bereits auf der Ebene der Problemdarstellung eine trügerische Verquickung von Grundlagenforschung und Anwendungsvision. Dies suggeriere eine nicht zutreffende Kontrollierbarkeit und Abschätzbarkeit möglicher Folgen und Entwicklungen. Schon hier ist es, so Esser, Aufgabe ethischer Reflexion, die Vorstellungen solcher bildsprachlichen Aussagen im Zusammenhang mit CRISPR-Cas aufzudecken und zu hinterfragen. Diese Überlegungen erfuhren im Publikum mehrfachen Widerhall und aktivierten ähnlich kritische Wortmeldungen, etwa zur pejorativen Rede von der „Eliminierung kranker Gene“.

Für einen interessanten Kontrast sorgte demgegenüber der anwendungsorientierte Blick Reinhard Merkels (Hamburg) auf das ethische Gebot der Behandlung schwerer Krankheiten sowie die Risiken der Risikovermeidung. Eine diskursive Vermittlung dieser Perspektiven gelang jedoch nicht. 
Noch grundsätzlicher war die Kritik Sigrid Graumanns (Bochum), die sich bei der Debatte an die ersten Auseinandersetzungen mit somatischen Gentherapien in den 1990er-Jahren erinnert fühlte. Heute wie damals sei eine grob vereinfachende Dualisierung von ,ethisch gutem“ Potenzial und „ethisch verwerflichen“ Nebenwirkungen der fraglichen Verfahren zu registrieren. Dabei gerate aus dem Blick, dass therapeutische Eingriffe in die menschliche Keimbahn im Grunde kein sinnvolles Anwendungsfeld von CRISPR-Cas darstellen. Bevor überhaupt zielgerichtet in einen Embryo eingegriffen werden könne, müsse dieser mittels PID auf seine Tauglichkeit für den Eingriffszweck selektiert werden. In diesem Zuge könne ebenso ein gesunder Embryo ausgewählt und übertragen werden. Dessen ungeachtet seien Vorstellungen vom einfachen Zusammenhang zwischen Genotyp und Phänotyp, von einer treffsicheren und vorhersehbaren Manipulation durch den präzisen Aus- und Einbau von Genbausteinen (,Designer-Babys“), schlicht Science Fiction.

In dieser konkreten Frage der Vorzugswürdigkeit von PID oder Keimbahneingriffen war die Rednerschaft in prägnanter Weise zweigeteilt. Während auf der einen Seite klinische Fälle geltend gemacht wurden, in denen die Vorschaltung einer PID aufgrund der hohen Anzahl betroffener Embryonen nicht sinnvoll sei (Merkel) und die Vermeidung von Embryonenselektion und -verwerfung als Vorzug der CRISPRCas-Methode herausgestellt wurde (Taupitz, Schockenhoff), führten Befürworter der PID die mögliche Folgenlast und „Totalität“ (Birnbacher) von Keimbahneingriffen für nachkommende Generationen an (Welte).

Mit den Beiträgen Carl Friedrich Gethmanns (Siegen) und Ingrid Schneiders (Hamburg) wurde ein abschließender Blick auf Prozesse der Schwellensenkung, Gefahren von Pfadabhängigkeit und „Locked-in“-Situationen - gewissermaßen auf die eingangs erwähnten „Fallen des Irrationalen“ - geworfen, die mit dem „schnell, einfach, kostengünstig und präzise“ (Vogel) funktionierenden CRISPR-Cas-Verfahren einhergehen können. Erlebt eine noch bis dato für unzureichend sicher befundene Technik einen sprunghaften Fortschritt, ist nach Gethmann Aufmerksamkeit beim Überschreiten moralischer Standards wie dem Prinzip der informierten Zustimmung, beim Übergang von Tier- auf Humanexperimente sowie bei Zieländerungen von therapeutischen auf eugenische Zwecke geboten. Eine solche Vorsichtshaltung kann, so Schneider, durchaus differenziert gestaltet werden. Denn technische Entwicklungen verliefen selten nach dem Muster eines linearen, unaufhaltsamen Dammbruchs, wenngleich Faktoren wie plötzliche Machbarkeit, Reputations-, Patent- oder Standortkonkurrenz bestimmte Entscheidungsprozesse beschleunigen könnten.

Den letzten Überlegungen kann sicherlich hinzugefügt werden, dass rasante Entwicklungen wie die der CRISPR-Cas-basierten „Genchirurgie“ auch einen Wandel gewohnter Diskursmuster beschleunigen können. Für den Jahrestag des Deutschen Ethikrats 2016 mag zu verzeichnen sein, dass die konkreten Positionen der Vertreter von nutzenorientierten Argumenten und der Vertreter einer deontologisch geprägten Sichtweise im derzeitigen Sachstand der Keimbahninterventionen praktisch konvergierten. Über einen vorsichtsbedingten Verzicht auf humane Keimbahninterventionen zu therapeutischen Zwecken herrscht zum gegenwärtigen Zeitpunkt weitgehende Einigkeit. In einen Streit gerieten dabei nicht biokonservative Pflicht- und bioliberale Abwägungsbegriffe. Die brisantere Frontenstellung ergab sich entlang der Frage nach dem richtigen Verständnis des eigentlichen Problems. Sind ethische Überle- 
gungen zu den möglichen Konsequenzen einer zukünftigen Technik zum jetzigen Zeitpunkt gerechtfertigt? Oder besetzt, wie Graumann und Esser andeuteten, die Stilisierung von CRISPR-Cas auf sein Anwendungspotenzial für Keimbahnkorrekturen lediglich den Platz des ethisch Verwerflichen, während andere Anwendungen im Licht des Unbedenklichen stehen? Um Dabrocks Metapher ein letztes Mal zu würdigen: Es scheint in der Natur von Schreckgespenstern zu liegen, dass über ihr Vorhandensein gestritten wird.

\section{Literatur}

Deutscher Ethikrat (2016) Zugriff auf das menschliche Erbgut. Neue Möglichkeiten und ihre ethische Beurteilung. http://www.ethikrat.org/veranstaltungen/jahrestagungen/zugriff-auf-das-menschlicheerbgut. Zugegriffen: 5. Sept. 2016

Liang P, Xu Y, Zhang X et al (2015) CRISPR/Cas9-mediated gene editing in human tripronuclear zygotes. Protein Cell 6(5):363-372. doi:10.1007/s13238-015-0153-5 